\title{
The mystery of the disappearing eye: A neonatal case report
}

\author{
L G Lloyd, MB ChB, DCH (SA), FCPaed (SA), MMed (Paed), Cert Neonatol (SA); \\ W de Witt, MB ChB, MMed (Paed), DTE \\ Department of Paediatrics: Neonatology, Steve Biko Academic Hospital and University of Pretoria, Pretoria, South Africa
}

Corresponding author: L G Lloyd (lizel.lloyd@up.ac.za)

Neonatal endophthalmitis is a rare but life-threatening condition that can have both endogenous and exogenous origins. Early diagnosis and aggressive treatment are vitally important to prevent death and disability, but mortality rates remain high. This case report demonstrates the rapid progression of the condition, which resulted in severe morbidity.

S Afr J CH 2014;8(1):34-36. DOI:10.7196/SAJCH.589

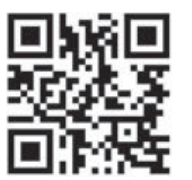

A male infant was born to a 30-year-old woman (para 2, gravida 3) via caesarean section for placenta praevia. Routine antenatal blood tests revealed the mother to be HIV-negative. She did not have syphilis, and her blood group was rhesus negative. She reported no problems during pregnancy other than the antepartum haemorrhage, after which the diagnosis of placenta praevia was made on antenatal ultrasound. This was managed conservatively with bed rest, and steroids were given to improve fetal lung maturity. The rest of the antenatal ultrasound examination was unremarkable. There was no history of any infections during the pregnancy.

The baby was delivered at a gestational age of 30 weeks according to the Ballard score and an early obstetric ultrasound scan, weighing $1700 \mathrm{~g}$ (between the 50th and 90th centiles), with a head circumference of $30 \mathrm{~cm}$ (on the 90th centile). The Apgar score was 7/10 at $1 \mathrm{~min}$ and $9 / 10$ at $5 \mathrm{~min}$. He was admitted to the neonatal intensive care unit at Steve Biko Academic Hospital, Pretoria, South Africa, for management of mild respiratory distress, for which he received only nasal prong oxygen at $1 \mathrm{l} / \mathrm{min}$. Antibiotic treatment with penicillin and amikacin was initiated. The full blood count (FBC) was normal, the C-reactive protein (CRP) level was $<1 \mathrm{mg} / \mathrm{l}$ (normal reference range $<10 \mathrm{mg} / \mathrm{l}$ ), and blood culture was negative.

On day 2 of life, the baby developed jaundice, for which phototherapy was initiated. He was transferred to the high-care unit, as he was clinically well and active.

Peri-orbital swelling of the left eye with mild discharge and red conjunctiva was noted on day 3 of life, and a clinical diagnosis of conjunctivitis was made by the consultant. Topical therapy with chloromycetin ointment was started in addition to the intravenous antibiotics initiated on day 1 of life. On day 4, blood investigations were repeated, revealing a raised CRP level of $16.6 \mathrm{mg} / \mathrm{l}$, with toxic granulation on the peripheral blood smear. A pus swab of the eye showed no growth.

On day 5, the baby deteriorated clinically, requiring more oxygen (2 1/min via nasal prongs). There was also a bloody discharge from the left eye. The orbit appeared hollow on day 6 , and on examination the eye could not be visualised (Fig. 1). An ophthalmology consultation was requested.

The ophthalmologists reported a perforated cornea, with swelling of the lens. The origin of the injury was thought to be a corneal abrasion secondary to the phototherapy mask, leading to endophthalmitis. Enucleation was the recommended treatment. Topical chloromycetin was continued, and the ophthmologists added ciprofloxacin eye drops. They also performed a second pus swab, which showed Gram-positive cocci but no growth. The findings on examination of the right eye were normal.

The FBC and tests for infective markers were repeated, and the CRP had increased to $56.3 \mathrm{mg} / \mathrm{l}$. Serological investigations for congenital infections excluded cytomegalovirus, herpes simplex virus, toxoplasma and rubella. The antibiotics were changed to meropenem, and vancomycin was added the next day to cover methicillin-resistant Staphylococcus aureus. Gonococcal conjunctivitis was considered, but thought to be unlikely as the delivery mode had been caesarean section, the condition was unilateral, and maternal history was negative.

The baby deteriorated clinically, and developed opisthotonus with increased tone in all four limbs with fisting. A cranial ultrasound scan and lumbar puncture were carried out. The ultrasound scan showed bilateral and symmetrical dilatation of the lateral ventricles with a hypo-echoic lesion in the left frontal lobe superior to the orbital part of the frontal bone. This was surrounded by more hyper-echoic cerebral parenchyma involving almost the entire frontal lobe on the left. Similar complicated cystic areas could be seen in the occipital lobes posterior to the posterior horns of the right and left lateral ventricles. An echogenic fluid collection measuring $1 \mathrm{~cm}$ was seen overlying the left parietal lobe. This was reported as a suspected subdural empyema (Figs 2 - 5).

The lumbar puncture revealed polymorphonucleocytes $30 \times 10^{9} / 1$, lymphocytes $86 \times 10^{9} / 1$, erythrocytes $3438 \times 10^{9} / 1$ and glucose $1.5 \mathrm{mmol} / \mathrm{l}$. The Gram stain revealed no organisms; bacterial antigen tests for group B streptococci (GBS), Haemophilus influenzae and Streptococcus pneumoniae were all negative; and the culture had no growth. The baby was discussed with the paediatric infectious diseases team, and the antibiotic treatment was deemed appropriate.

By day 10, the CRP level had normalised to $<1 \mathrm{mg} / \mathrm{l}$. On day 12 , the baby improved clinically, and the enucleation was performed on day 13 . The surgery was attended by a microbiologist, and immediate Gram staining was negative. The specimen showed no bacterial or fungal growth, and neutrophils $3+$ were present.

Although the patient improved clinically, there was irreversible brain damage with progressive worsening on ultrasound and computed tomography (CT) images (Figs 6 - 8).

A ventriculo-peritoneal shunt was inserted after the CT scan. The baby has severe neurodevelopmental delay. He received an ocular implant just before his first birthday, and is being followed up for neurodevelopmental intervention.

\section{Discussion}

Invasive bacterial eye infections in the neonate are a rare but serious condition. ${ }^{[1,6]}$ Mortality rates are reportedly high and visual prognosis 


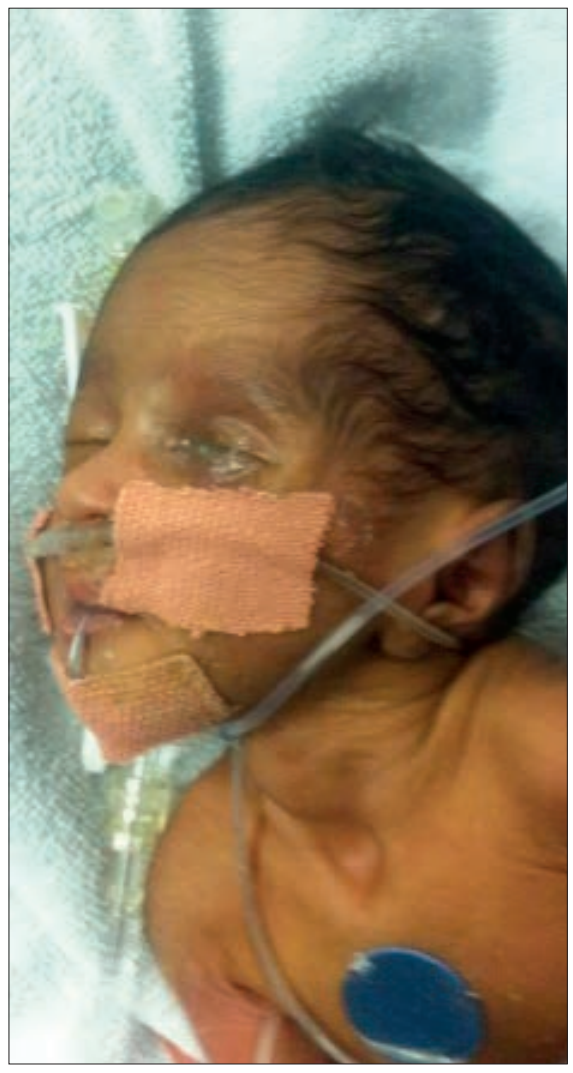

Fig. 1. The patient on day 7 after birth, illustrating the left orbit (reproduced with the permission of the parents).

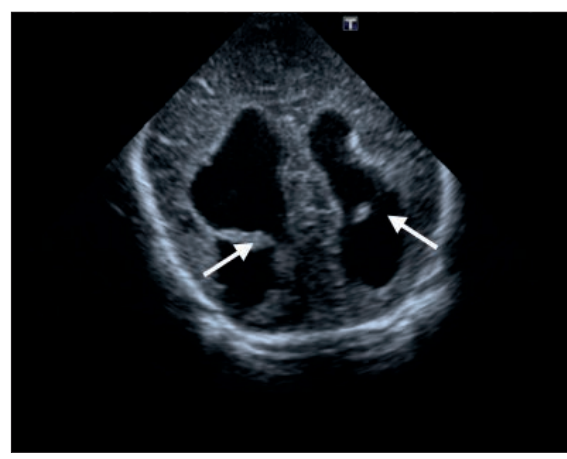

Fig. 6. Day 26. Both posterior abscesses have broken through to the posterior horns of the ventricles (arrows).

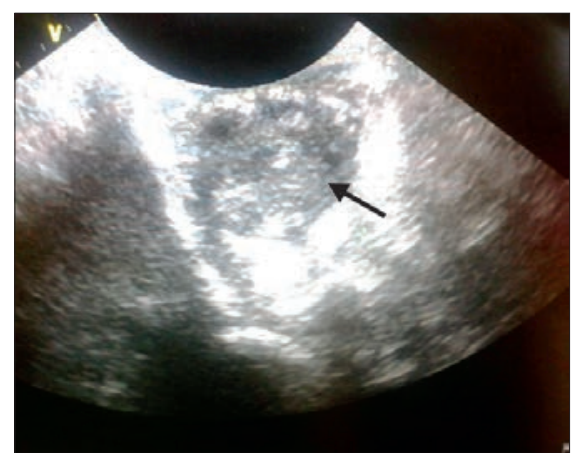

Fig. 2. Ultrasound image of the abnormal left orbit (arrow). The orbit could not be demonstrated owing to tissue destruction (compare with Fig. 3).

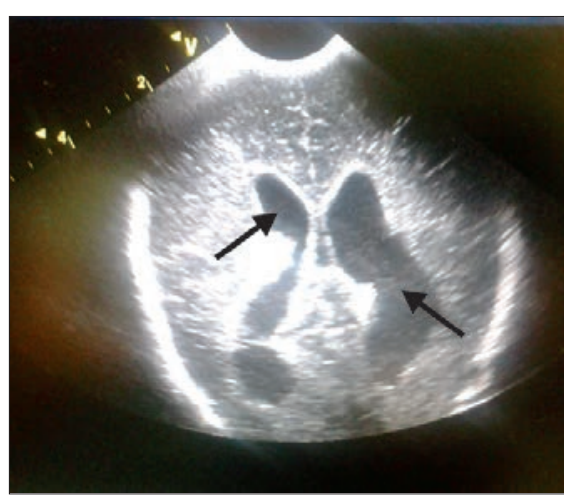

Fig. 4. Coronal view of the brain, revealing dilated lateral ventricles (arrows)

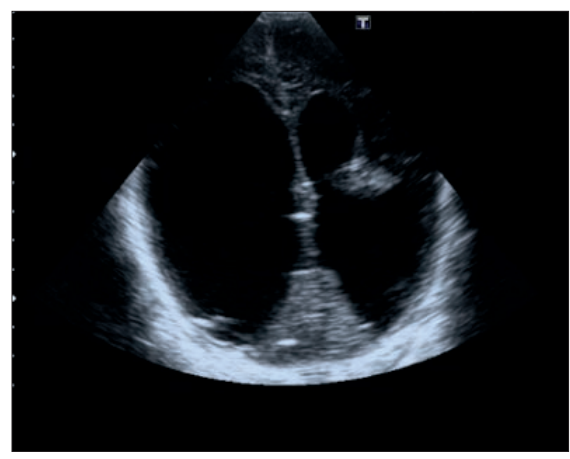

Fig. 7. Two months. There is massive dilatation of the ventricles with porencephalic cysts.

injury or intra-ocular surgery can result in exogenous endophthalmitis. ${ }^{[4,5,7]}$

Pseudomonas aeruginosa is reported to be the organism most frequently cultured (in up to $80 \%$ of cases). ${ }^{[4]}$ The source may be endogenous, secondary to septicaemia, or nosocomial, associated with contaminated humidifiers, suction apparatus, incubators and ventilators. ${ }^{[1,2]}$ Pseudomonas is a very virulent organism, exerting its effect by the production of proteoglycanolytic enzymes, as well as the production of endo- and exotoxins. These contribute to the local breakdown of

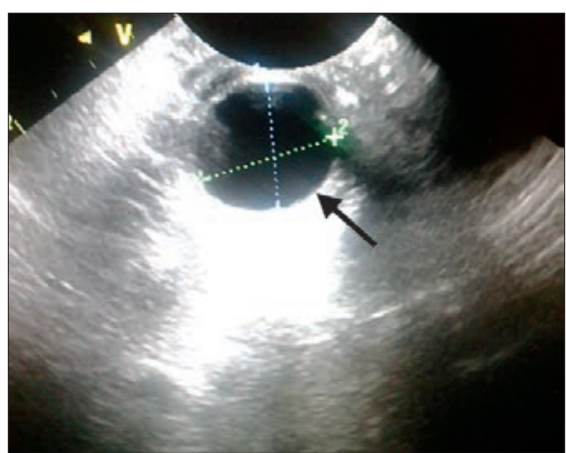

Fig. 3. Ultrasound image of the normal right orbit (arrow).

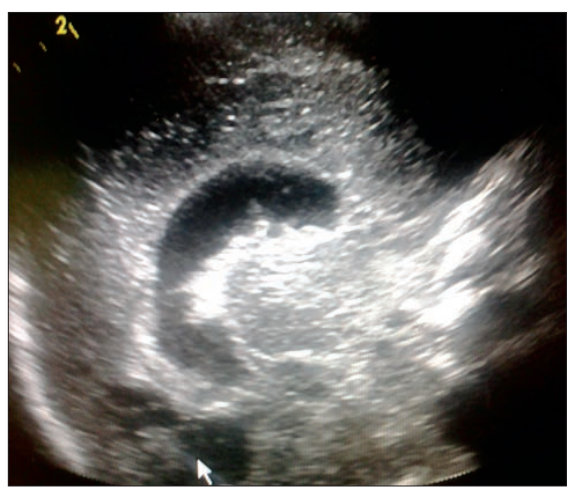

Fig. 5. Sagittal image with complicated cystic areas posterior to the posterior horns of both lateral ventricles (arrow)

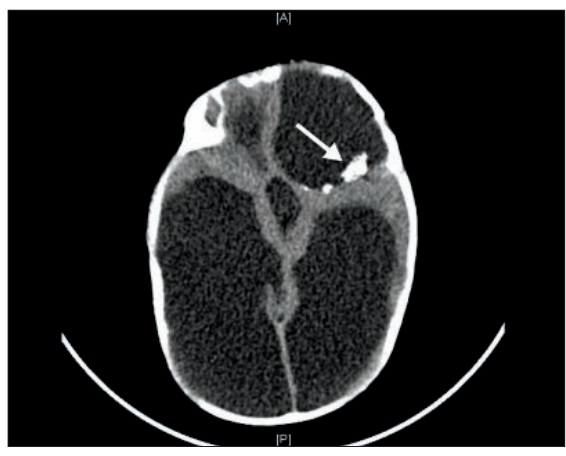

Fig. 8. Three months. Obvious macrocephaly with massively dilated lateral and third ventricles. Calcifications (arrow) are noted along the horn of the left frontal horn. There is an obstructive hydrocephalus at the level of the fourth ventricle.

tissue defences, including corneal stroma and conjunctival blood vessels. ${ }^{[2,4]}$

GBS have been described, especially in premature infants with reduced levels of maternal antibodies. ${ }^{[4]}$ Other organisms identified include Salmonella enteritidis, $H$. influenzae type $\mathrm{b}$ and meningococci. ${ }^{[1,4]}$ In earlier reports, $S$. aureus was associated, but it is not mentioned in more recent literature. ${ }^{[1]}$ Viruses, fungi and protozoa can also cause endophthalmitis, especially Candida. ${ }^{[1]}$

The initial presenting symptom is purulent conjunctivitis in $>80 \%$ of cases. ${ }^{[1,5]}$ Symptoms 
include eyelid swelling and erythema, corneal oedema and infection, injected conjunctiva and sclera, purulent discharge, hypopyon, vitreitis, vitreal mass and debris, reduced or absent red reflex, limited view of the fundus and proptosis. ${ }^{[3,4]}$ The differential diagnosis of leucocoria, which can be a presenting symptom, includes cataract, retinoblastoma or retinal detachment. ${ }^{[3]}$ Meningitis and endotoxic shock are known complications of neonatal conjunctivitis. ${ }^{[2,5]}$

Pseudomonas infection can have a fulminant course, rapidly progressing from conjunctivitis to perforating keratitis to endophthalmitis within 72 hours. ${ }^{[1]}$ Corneal destruction seems to be mediated by a proteoglycanolytic enzyme capable of degrading corneal ground substance rapidly. It is complicated with septicaemia in $>50 \%$ of cases, diagnosed on average 5 days after the onset of conjunctivitis, and fatal in $40 \%$ of cases. ${ }^{[1]}$ Bilateral endophthalmitis can complicate neonatal pseudomonal infection, and is a grave sign for survival ${ }^{[8]}$

As there are several physiological conjunctival flora, and as these organisms are often replaced by nosocomial flora, conjunctival swabs are not a reliable diagnostic tool. ${ }^{[1]}$ However, growth of $P$. aeruginosa on such a swab should be viewed as indicating a possible lifethreatening infection. ${ }^{[4]}$ Material obtained from the anterior chamber or a vitreous tap is more specific. ${ }^{[1]}$ This procedure should only be performed by an experienced ophthalmologist, and is therefore not practical. ${ }^{[1]}$ Blood culture has been reported to be positive in $>90 \%$ of cases, with vitreal cultures positive in only $56 \%{ }^{[3]}$ As there is no consensus in the literature regarding the best diagnostic tool, the combination of Gram stains and blood cultures seems to be of most diagnostic value in invasive bacterial eye infections. ${ }^{[1]}$

The usefulness of topical antibiotics is limited by poor penetration into the anterior chamber and vitreous humour. ${ }^{[1]}$ A combination of topical and systemic antibiotics has not been proven to be superior to systemic antibiotics alone. ${ }^{[1]}$

Early systemic antibiotics remain the cornerstone of treatment. ${ }^{[3]}$ However, the use of parenterally administered cephalosporins that are effective against Pseudomonas is recommended. ${ }^{[1]}$ In the case of GBS infections, penicillin $\mathrm{G}$ and an aminoglycoside are advised. ${ }^{[1]}$ Intraocular injection of antibiotics into the anterior chamber or the vitreous humour should be reserved for 'desperate cases' since there is little experience and few data on potential toxicity. ${ }^{[1]}$ Intravenous vancomycin in combination with a third-generation cephalosporin or aminoglycoside may also be used. ${ }^{[4]}$

Pseudomonas infection has a high mortality rate of $40 \%$, with all surviving patients blind or severely visually impaired in the affected eye. ${ }^{[1,3,5]}$ GBS endophthalmitis seems to have a more favourable prognosis, with partial preservation of function of the eye. ${ }^{[1]}$

\section{Conclusion}

Our patient received early aggressive antibiotic treatment, to which he responded, but unfortunately the neurological damage was irreversible. Cultures, including cerebrospinal fluid, were negative throughout, making it difficult to adjust treatment. Although we never proved that Pseudomonas infection was present, we had one other patient in the ward at that time with a blood culture positive for P. aeruginosa.

Neonatal conjunctivitis is a common condition that is often regarded as harmless. We would like to emphasise the importance of thorough ocular examinations in all neonates to enable early diagnosis and management, and hopefully prevention, of possible life-threatening complications.

Acknowledgements. We thank the patient's parents for allowing the images to be published, Dr H M Swanepoel for the ultrasound images, Prof. T Avenant and the infectious diseases team for their input, and all the doctors involved in the management of this case.

\section{References}

1. Lohrer R, Belohradsky BH. Bacterial endophthalmitis in neonates. Eur Pediatr 1987;146(4):354-359. [http://dx.doi.org/10.1007/BF00444936]

2. O'Keefe M, Nolan L, Lanigan B, Murphy J. Pseudomonas aeruginosa endophthalmitis in a preterm infant. J AAPOS 2005;9(3):288-289. [http:// dx.doi.org/10.1016/j.jaapos.2005.01.012]

3. Sparks JR, Recchia FM, Weitkamp JH. Endogenous group B streptococcal endophthalmitis in a preterm infant. J Perinatol 2007;27(6):392-394. [http:// dx.doi.org/10.1038/sj.jp.7211685]

4. Figueiredo S, João A, Mateus M, Varandas R, Ferraz L. Endogenous endophthalmitis caused by Pseudomonas aeruginosa in a preterm infant: A case report. Cases J 2009;2(1):9304. [http://dx.doi.org/10.1186/1757-1626-2 9304]

5. Gaili H, Woodruff GHA. Exogenous Pseudomonas endophthalmitis: A cause of lens enucleation. Arch Dis Child Fetal Neonatal Ed 2002;86(3):F204-F206. [http://dx.doi.org/10.1136/fn.86.3.F204]

6. Toshiaki K, Yayoi S, Junko W, et al. Corneal perforation in a premature infan at the corrected gestational age of 32 weeks. Japanese Journal of Clinica Ophthalmology 2004;58(2):171-173.

7. Tovilla-Canales JL, Nava A, Tavilla y Pomar JL. Orbital and peri-orbital infections. Curr Opin Ophthalmol 2001;12(5):335-341. [http://dx.doi. org/10.1097/00055735-200110000-00002

8. Boyle EM, Ainsworth JR, Levin AV, Campbell AN, Watkinson M. Ophthalmic Pseudomonas infection in infancy. Arch Dis Child Fetal Neonatal Ed 2001;85(2):F139-F140. [http://dx.doi.org/10.1136/fn.85.2.F139]

9. For T, Wong W, Chang A. Use of eyepatches in phototherapy: Effects on conjunctival bacterial pathogens and conjunctivitis. Pediatr Infect Dis 1995;14(12):1091-1094. [http://dx.doi.org/10.1097/00006454-19951200000012] 